\title{
REDUÇÃO DA PREVALÊNCIA DE ÚLCERA DUODENAL: um estudo brasileiro (análise retrospectiva na última década: 1996-2005)
}

\author{
Carlos SAUL ${ }^{1}$, Cláudio Rolim TEIXEIRA², Julio C. PEREIRA-LIMA³ e Ronaldo J. Spinatto TORRESINI²
}

RESUMO - Racional - A úlcera duodenal sempre representou uma doença muito prevalente entre as enfermidades digestivas, em qualquer parte do mundo. A prevalência média era de aproximadamente $10 \%$ da população mundial. A partir do início dos anos 90 , a literatura, tanto européia como norte-americana, passou a demonstrar sua redução gradativa entre seus países. Objetivo - Demonstrar, através de análise retrospectiva, a prevalência anual da úlcera duodenal nos últimos 10 anos em um Serviço de Endoscopia Digestiva que é referência para o sistema público de saúde da cidade de Porto Alegre, municípios da Grande Porto Alegre e outras cidades vizinhas da mesma. Os dados analisados são de março de 1996 até dezembro de 2005. Métodos - Estudo retrospectivo transversal, com análise documental de diagnósticos endoscópicos efetuados em endoscopia digestiva alta, no referido Serviço. Foi feita a análise retrospectiva de diagnósticos endoscópicos efetuados em 13.130 pacientes submetidos a endoscopia digestiva alta no período de março de 1996 a dezembro de 2005. A classificação de Sakita foi utilizada para o estádio do grau evolutivo da úlcera duodenal e foi considerado por ela acometido o paciente com a lesão no estágio A1, até o estágio S1, inclusive. Observou-se também a prevalência nos dois sexos, na raça, o percentual médio total nos 10 anos, além da prevalência anual. Para verificar se houve significância estatística dos resultados observados nos diferentes períodos, foi aplicado um teste de regressão linear ("linear regression model"). Resultados - Observou-se decréscimo gradativo dos percentuais de prevalência da úlcera duodenal, ano após ano, iniciando-se em 1996 com 8,6\% e se encerrando no final de 2005, com 3,3\%. A exceção do período foi observada no ano de 2003 quando houve um acréscimo, comparando-se com o decréscimo gradativo dos 6 anos anteriores. Mas já, a partir do período seguinte (2004), a queda gradativa voltou a ser observada. O valor médio de queda anual, aplicando o teste de regressão linear, foi da ordem de 1,3\% ao ano, no período analisado (10 anos). Este teste mostrou também significância estatística. A raça branca representou a maioria, com $78 \%$ dos pacientes, em relação a raça negra. O sexo masculino, em todos os períodos analisados, exceto em um (1997), foi o sexo mais acometido, com uma relação final masculino/feminino da ordem de 1.17/1.0. Conclusões - Observou-se então, neste estudo efetuado no Brasil, também uma redução da prevalência da úlcera duodenal, já apontada pela literatura em outros países. Estudos posteriores devem ser efetuados no sentido de apontar as razões desta importante observação.

DESCRITORES - Úlcera duodenal, epidemiologia.

\section{INTRODUÇÃO}

A úlcera duodenal (UD) sempre representou um diagnóstico endoscópico de alta freqüência em qualquer serviço de endoscopia digestiva. Por mais de um século a doença ulcerosa péptica foi manejada cirurgicamente com médias de morbidade e mortalidade importantes ${ }^{(30)}$. Nas duas últimas décadas, importantes mudanças têm ocorrido na epidemiologia dessa doença ${ }^{(17)} \mathrm{e}$, a partir de 1996, a literatura passou a relatar gradativa diminuição da sua prevalência.

A supressão farmacológica efetiva da acidez gástrica começou com a introdução no mercado dos antagonistas do receptor $\mathrm{H} 2$ da histamina nos anos 70 , com resultados clínicos de grande melhora. Durante os anos 80 , a cirurgia eletiva para a úlcera péptica declinou em $85 \%$, o que pode ser atribuído principalmente ao uso desses antagonistas ${ }^{(6)}$. No final dos anos 80 surgiram os inibidores da bomba de prótons (IBP) cujo primeiro representante foi o omeprazol. Com o desenvolvimento destes, a ainda maior redução da secreção gástrica e a não ocorrência de taquifilaxia no tratamento com estes IBP, veio assegurar mais altos índices ainda na cicatrização da doença ulcerosa ${ }^{(20)}$.

A prevalência da úlcera difere ao redor do mundo com a UD predominando nos países ocidentais e as úlceras gástricas (UG) sendo mais freqüentes na Ásia, especialmente no Japão ${ }^{(23)}$. Embora a incidência da doença ulcerosa péptica nos países ocidentais tenha declinado nos últimos 100 anos, 1 de cada 10 americanos continuavam a ser por ela afetados ${ }^{(24)}$.

${ }^{1}$ Faculdade de Medicina, Universidade Federal de Pelotas, RS; ${ }^{2}$ FUGAST (Fundação Riograndense Universitária de Gastroenterologia), Porto Alegre, RS; ${ }^{3}$ Faculdade de Medicina da Fundação Federal Faculdade de Ciências Médicas, Porto Alegre, RS

Correspondência: Dr. Carlos Saul - Rua Silva Só, 255 - Bairro Santa Cecília - 90610-270 - Porto Alegre, RS. E-mail: csaull@yahoo.com.br 
O isolamento do Helicobacter pylori (H. pylori) e sua identificação como a causa mais importante na gênese da úlcera péptica levou a exploração do papel da inflamação, e sua associada cascata de citoquinas, na secreção ácida gástrica ${ }^{(30)}$.

A hipersecreção gástrica associada à síndrome de ZollingerEllison, a hiperplasia de células G, o aumento na massa de células parietais e o equilíbrio fisiológico entre hormônios gastrina e somatostatina são ainda importantes fatores a serem considerados na doença ulcerosa ${ }^{(30)}$. O estresse emocional, fumo, consumo de álcool, uso de antiinflamatórios-não-hormonais (AINES) incluindo o ácido acetil salicílico (AAS), cloreto de potássio, medicamentos imunossupressores, outras drogas, e a queda dos níveis de prostaglandinas relacionados com a idade, têm mostrado também serem fatores contribuintes no aparecimento da úlcera ${ }^{(29)}$.

Porém, muitos pacientes com doença ulcerosa não complicada são assintomáticos ou tratados empiricamente sem confirmação diagnóstica $^{(7)}$, e esse aspecto dificulta substancialmente estudos epidemiológicos da doença não complicada e pode ser o motivo de os estudos sobre a incidência, serem poucos ${ }^{(7)}$.

O objetivo do presente estudo foi verificar se, também em nosso meio, houve modificações na prevalência da úlcera duodenal.

\section{MÉTODOS}

Foi feita análise retrospectiva dos resultados de exames endoscópicos (endoscopia digestiva alta - EDA) de pacientes oriundos exclusivamente de serviços do Sistema Único de Saúde atendidos na Unidade de Endoscopia Digestiva do convênio FUGAST/Hospital Sanatório Partenon - Secretaria Estadual da Saúde e Meio Ambiente do Estado do Rio Grande do Sul, localizado em Porto Alegre.

Treze mil e cento e trinta pacientes foram examinados nessa unidade nos últimos 10 anos, no período compreendido de março de 1996 a dezembro de 2005 e foram o objeto do presente estudo. Os pacientes tinham origem de ambulatório (94\%), oriundos de Postos de Saúde Pública de Porto Alegre, Municípios da Grande Porto Alegre e de algumas cidades vizinhas, e de internação hospitalar $(6 \%)$ no Hospital Sanatório Partenon de Porto Alegre. Os exames de EDA foram todos executados ou observados diretamente pelo mesmo examinador (C.S.).

Foram considerados com UD os pacientes cujo exame endoscópico demonstrou a presença de lesão duodenal nos estágios A1 a S1 da classificação de Sakita ${ }^{(21)}$, classificação esta de conhecimento amplo e de fácil entendimento. Videoendoscópios da marca Fujinon, modelo $250 \mathrm{~S}$ e seus acessórios, foram utilizados.

Os resultados dos exames endoscópicos ficam registrados em arquivo informatizado, onde também está arquivado o consentimento informado prestado por cada paciente precedendo o exame. Foram também observados o sexo e a raça dos pacientes (branca ou negra). Não foi feito inquérito a respeito do uso de AINES ou outras medicações, como também não foi observada a presença ou não do $H$. pylori, pois não eram os objetivos do presente estudo, uma vez que este teve como alvo precípuo a pesquisa da prevalência da UD no período analisado.

Para verificar se houve significância estatística dos resultados obtidos, utilizou-se um modelo de regressão linear ("linear regression test"), onde é verificado se a inclinação do modelo linear obtido é diferente de uma declinação nula $(b=0$, hipótese nula).

Esta pesquisa foi submetida a apreciação do Comitê de Ética em Pesquisa da Faculdade de Medicina da Universidade Federal de Pelotas, RS, e por ele aprovada.

\section{RESULTADOS}

Treze mil e cento e trinta pacientes foram submetidos a EDA no período de março de 1996 a dezembro de 2005. No ano de 1996 foram examinados 947 pacientes e em 79 deles observou-se UD, com percentual de prevalência de $8,3 \%$. No ano de 1997 foram 1.436 pacientes com 87 casos de UD, num percentual de 6,0\%. Já em 1998, 1.609 foram examinados, com $4,2 \%$ de prevalência, que correspondeu a 68 pacientes. No ano seguinte, 1999, foram feitas 1.543 EDA e em 5,7\% dos pacientes havia UD, totalizando 88 casos. Em 2000, 1.261 pacientes foram examinados e em 70 deles havia UD, correspondendo a 5,5\% de percentual. No ano de 2001, 1.175 pacientes submeteram-se a EDA e em $4,1 \%$ deles, ou seja, em 48 pacientes, havia UD. Um mil e cento e vinte e dois pacientes foram examinados em 2002 e 40 deles tinham UD, com percentual de 3,9\%. No ano de 2003 observou-se acréscimo de casos de UD em relação aos 6 anos anteriores, atingindo-se o percentual de $6,5 \%$, que correspondeu a 85 pacientes, em 1.305 examinados. Em 2004, 1.400 pacientes foram examinados e em $3.9 \%$ deles, ou seja, em 55 pacientes, foi evidenciada UD. Por fim, no ano de 2005, dos 1.372 exames efetuados, em 46 casos havia a lesão duodenal, que correspondeu a $3,3 \%$ dos pacientes examinados. A Figura 1 mostra esta distribuição.

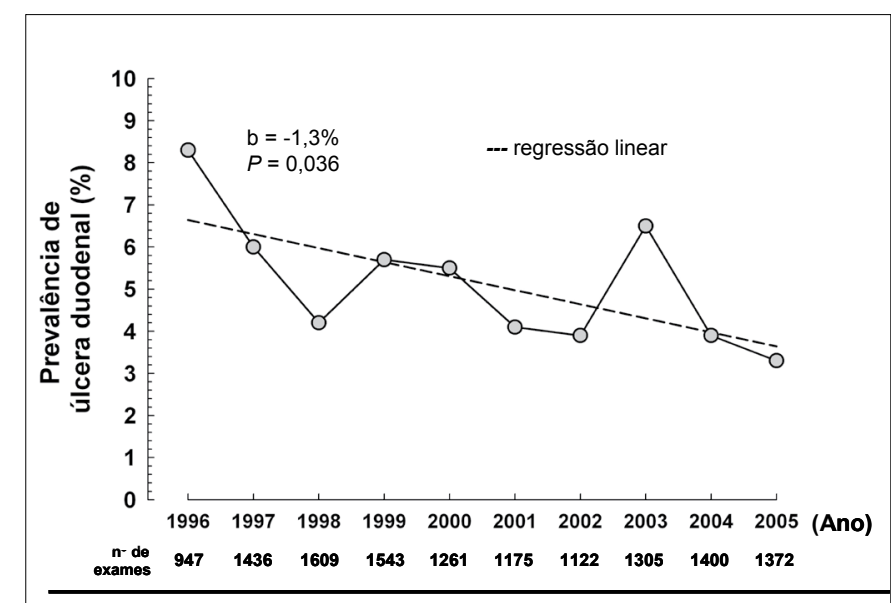

FIGURA 1. Gráfico de linha representando a queda na prevalência de úlcera duodenal observada em exames endoscópicos, FUGAST/Hospital Sanatório Partenon, Porto Alegre, RS 
Observou-se então, no transcorrer dos 10 anos que abrangeu o estudo, queda progressiva na prevalência da UD, de forma seqüencial, não homogênea e consecutiva, excetuando-se o ano de 2003 quando houve crescimento em relação ao ano anterior, mas já no ano seguinte (2004) voltou a cair. A prevalência no primeiro ano da década estudada foi de $8,3 \%$ e a prevalência observada no último ano de abrangência do estudo foi de $3,3 \%$. Colocados os resultados no modelo de regressão linear empregado, este teste mostrou uma relação de queda $(b=-1.3)$ da taxa de prevalência anual da UD. A redução média anual, calculada no período pelo teste empregado ("linear regression model") foi de $1,3 \%(b=-1.3)$, com significância estatística apontada $(P=0,036)$.

Em relação à idade dos pacientes, o mais jovem com UD tinha 16 anos e o mais idoso 71, e a faixa de idade de maior acometimento registrada foi dos 35 aos 50 anos.

Quanto ao sexo dos pacientes, observou-se em todos os períodos anuais analisados, exceto um (1997), predomínio do sexo masculino e a correlação final da prevalência nos sexos foi 1,17 homens para 1,0 mulheres (M 1,17/1,0 F).

\section{DISCUSSÃO}

Nos anos 60, SUSSER e STEIN ${ }^{(25)}$ foram os primeiros a sugerir o declínio na incidência de úlcera péptica baseados em estudo de coorte analisando mortalidade. Diversos estudos dos últimos anos têm apontado para a queda da prevalência da úlcera péptica, como também de cirurgias realizadas para o tratamento desta doença e também das internações hospitalares e cirurgias para solucionar suas complicações. Autores demonstraram redução de $80 \%$ do número de cirurgias do trato digestivo alto, tanto por intratabilidade, como por complicações como sangramento, obstrução e perfuração ${ }^{(23)}$. Outro estudo realizado na Holanda, no período 1992-2003, mostrou que caiu pela metade o número de casos com úlcera gástrica ${ }^{(17)}$. Também as internações por úlcera péptica diminuíram consideravelmente entre 1980 e 2003, mas não mudou ou subiu levemente, o número de casos de úlceras complicadas ${ }^{(17)}$. Outro estudo mostrou que em pacientes homens com UG, em 1980, havia 32 casos por 100.000 e no ano de 2003 este número caiu para 16 por 100.000. Entre as mulheres registraram-se 21 casos por 100.000 em 1980 e 15 por 100.000 em $2003^{(30)}$. Já entre os pacientes com UD, observaram-se 51 casos por 100.000 em 1980, entre os homens, que caiu para 28/100.000 no ano de 2003. Entre as mulheres, em 1980, registraram-se 20 casos por 100.000 e no ano de 2003 caiu para 12 por $100.000^{(30)}$.

No presente estudo observou-se, no período analisado de 10 anos, redução gradativa, não homogênea e seqüencial, do percentual de prevalência anual da UD. Assim, no período 1996-1997 a queda percentual foi de $2,3 \%$ em relação ao período anterior e a queda foi de 1,8\% no período seguinte (1997-1998). Nos outros períodos, as quedas foram menores, em torno de $0,5 \%$, um pouco acima, um pouco abaixo deste valor, mas sempre com registro de queda. A exceção observada no presente estudo ocorreu no período 2003-2004, quando se registrou aumento de prevalência em relação ao período anterior, mas já no período seguinte ao estudo (2004-2005) voltou a se observar sua queda. O registro da prevalência observada no primeiro dos 10 anos do estudo foi de $8,3 \%$ e a prevalência registrada ao final do mesmo foi de 3,3\%. O modelo estatístico empregado de regressão linear mostrou inclinação no módulo de teste de $1.3(\mathrm{~b}=-1.3)$, que se traduz como uma redução média anual da ordem de $1,3 \%$ e que demonstrou significância estatística $(P=0,0036)$. O registro isolado de aumento de prevalência em um único período, contrapondo-se ao registro de queda em todos os outros períodos, pode ter explicação no aspecto multifatorial da etiologia da UD e que alguns destes fatores causais pudessem ter influído no valor diferente observado em um único período. Mas no presente estudo não foi pesquisada a presença ou não destes fatores, pois o estudo direcionou-se somente no registro de prevalências em um período determinado.

Estudo realizado na Inglaterra e no País de Gales, no período 1994-1998, mostrou redução da prevalência da úlcera péptica, sendo que em homens a queda foi de 3,3 para 1,5/1000 e nas mulheres de 1,8 para $0,9 / 1000^{(9)}$.

Fumo, álcool, estresse, uso de medicamentos, faixa etária, infecção pelo $H$. pylori, hereditariedade de afecções pépticas, entre outros, são alguns dos fatores que podem desempenhar diferentes papéis na gênese da doença e, segundo a literatura, entre as principais hipóteses para esta queda de prevalência, está a maior eficácia do tratamento da UD e UG obtidas com a erradicação do $H$. pylori e o uso dos IBP. Autores referem que os mais importantes fatores de risco para a úlcera péptica são a infecção da mucosa gástrica pelo $H$. pylori e o uso de AINES ${ }^{(2)}$. Estudo desenvolvido na Austrália, que também apontou a redução da prevalência da úlcera péptica na última década, cita que as possíveis causas seriam o declínio da infecção pelo $H$. pylori e a redução no uso de $\operatorname{AINES}^{(28)}$. Outro estudo apontou a redução da prevalência da úlcera péptica como conseqüência do uso de bloqueadores $\mathrm{H} 2$, de inibidores da bomba de prótons, da introdução de AINES seletivos e a diminuição da infecção pelo H. pylori ${ }^{(30)}$. Estudo efetuado na Dinamarca aponta o hábito do fumo e a infecção pelo $H$. pylori como os maiores fatores de risco para a úlcera péptica ${ }^{(19)}$.

Mecanismos fisiopatológicos diferentes de lesão da mucosa determinam o aparecimento da UD e da UG. UD é essencialmente doença relacionada com o $H$. pylori e causada principalmente por aumento na secreção de ácido e pepsina, e metaplasia gástrica na mucosa duodenal $^{(4)}$. UG, principalmente em países do ocidente, está mais freqüentemente associada ao uso de AINES, embora a infecção pelo $H$. pylori também possa estar presente ${ }^{(10)}$. Gastrite crônica superficial ou atrófica predominam em pacientes com UG e mesmo níveis normais de secreção ácida podem estar associados à ulceração mucosa ${ }^{(27)}$.

Durante os anos 80, a infecção pelo $H$. pylori foi demonstrada em mais de $90 \%$ dos pacientes com UD e em torno de $70 \%$ dos pacientes com $U^{(15,5)}$. O declínio na incidência e prevalência da úlcera péptica em países desenvolvidos tem ocorrido em paralelo à queda da prevalência da infecção gástrica pelo $H$. pylori, principalmente em populações com altas médias de infecção ${ }^{(16,28)}$. O uso de AINES, a despeito de seu bem determinado efeito analgésico e antiinflamatório, é provavelmente a causa mais comum de 
lesão da mucosa gastrointestinal em países do ocidente. AINES, incluindo o AAS, aumentam significativamente o risco de eventos adversos gastrointestinais, em particular aqueles relacionados com a lesão da mucosa gástrica ou duodenal, na forma de erosões, úlceras e complicações de úlcera, especialmente sangramento ${ }^{(11)}$. Em torno de $15 \%$ a $30 \%$ dos usuários regulares de AINES, têm uma ou mais úlceras quando examinados periodicamente, e $3 \%$ a $4 \%$ destes têm sintomas gastrointestinais altos, úlcera ou complicações da mesma ${ }^{(30)}$. A infecção pelo H. pylori e o uso de AINES são fatores de risco independentes para úlcera péptica, mas com efeitos aditivos ou sinérgicos ${ }^{(30)}$. Em uma metanálise, a razão de chance de incidência de úlcera péptica foi de $1.1 \mathrm{em}$ pacientes infectados com H. pylori e também usando AINES ${ }^{(8)}$. Esta chance subiu marcadamente para 18.1 quando pacientes com $H$. pylori foram comparados com pacientes sem $H$. pylori e não em uso de AINES. A infecção pelo $H$. pylori também potencializa o sangramento da úlcera induzido por uso de AAS de baixa dose ${ }^{(12)}$. Juntos, o H. pylori e os AINES, concorrem para o aparecimento de $90 \%$ das úlceras pépticas ${ }^{(30)}$.

Um estudo (Funen County) demonstrou o número de pacientes com úlcera péptica, complicada ou não, pela primeira vez, com ou sem prescrição de AINES, nos últimos 30, 90 ou 180 dias $^{(13)}$. Seus resultados demonstraram que entre 1993 e 2002 a incidência da úlcera não complicada como também da úlcera perfurada diminuiu, a incidência de úlcera com sangramento se manteve constante e verificou-se proporção aumentada na incidência da úlcera em pacientes em uso de AINES ${ }^{(13)}$. Autores referem que a diminuição da incidência da UG se baseia em dois fatores principais: a melhora das condições de vida e a queda na prevalência da infecção pelo $H$. pylori e que ambas vêm ocorrendo desde o final da Segunda Guerra Mundial ${ }^{(14,18)}$. POST et al..$^{(17)}$ apontam que a introdução da terapia para erradicação do $H$. pylori tem presumidamente reduzido ainda mais a prevalência da doença péptica, minimizando o risco de recidiva da úlcera em pacientes com dispepsia, mas que a maior contribuição na redução da incidência da doença ulcerosa veio com a introdução e o conseqüente uso dos IBP por todo o mundo. Estudo efetuado em uma população chinesa mostrou aumento do uso anual de drogas anti-úlcera na população geral, crescendo da prevalência de $9,6 \%$ em 1997, para $15,9 \%$ em $2001^{(3)}$. Em estudo de coorte recente, incluindo 600.000 pacientes com cuidados primários da saúde, foi verificado que a prevalência do uso de IBP aumentou de $2,5 / 100 /$ ano $(95 \%$ IC $2,4-2,7)$ em 1996, para 5,8/100/ano $(95 \%$ IC $5,6-5,9)$ em $2003^{(17)}$. Van LEERMANN et al. ${ }^{(26)}$ referem que as admissões hospitalares, tanto para UD como para UG diminuíram, demonstrando redução de $23 \%$ na prevalência de hemorragia digestiva alta no período 1993-2000 da ordem de 61,7/100.000 para 44,7/100.000 e que a metade daqueles pacientes havia ingerido AINES. Outro artigo mostra a redução, pela metade, do número de casos de úlcera péptica perfurada nos últimos anos ${ }^{(22)}$, mas estudo realizado na Polônia não mostrou mudanças na incidência de suas complicações, apesar da redução da infecção pelo $H$. pylori $i^{(1)}$.

\section{CONCLUSÕES}

Os registros por todo o mundo tendem a mostrar importante redução da prevalência da doença péptica. $\mathrm{O}$ presente estudo também demonstrou esta queda. Maiores estudos são necessários para comprovação das causas desta redução. Assim também, é esperado que esta doença possa seguir com prevalência importante tendo significante impacto global nos cuidados da saúde, nos gastos de recursos com a mesma e na qualidade de vida dos pacientes, pois a doença pode aumentar com o avanço da idade dos doentes, uma vez que a média de longevidade está crescendo em quase todo o mundo.

Saul C, Teixeira CR, Pereira-Lima JC, Torresini RJS. Prevalence reduction of duodenal ulcer: a Brazilian study. (Retrospective analysis in tha last decade: 1996-2005). Arq Gastroenterol. 2007;44(4):320-4.

ABSTRACT - Background - The duodenal ulcer always represented a very prevalent pathology among the gastrointestinal tract diseases worldwide. The average prevalence is approximately $10 \%$ of the world population. In the 90 s the literature (both European and North American) begin to show a crescent reduction of this prevalence in many countries. Aims - To show through a retrospective analysis the annual prevalence of the duodenal ulcer in the last 10 years in a Digestive Endoscopy Service which is referred to public medical system in Porto Alegre and its suburban area and countryside of the State of Rio Grande do Sul, Brazil. The data analyzed is from March 1996 to December 2005. Specific data: transversal retrospective study with documented analysis of endoscopic diagnosis of upper gastrointestinal endoscopy. Methods - A retrospective analysis of the diagnosis of the 13.130 procedures of upper gastrointestinal endoscopy between 1996 and 2005. The Sakita classification was used to verify the duodenal ulcer activity taking into consideration the patients who have lesions on A1 to S1 levels. To verify if there was a statistical significant results, a linear regression test was done (linear regression model). Results - A gradate decrease of the prevalence percentuals was observed, year after year, it began with $8.3 \%$ of prevalence in 1996 and finished with 3.3\% in the beginning of 2006 . The average annual reduction of this prevalence was calculated following the regression test and it was placed in the $1.3 \%$ a year in the studied period of time. In 2003, in an isolated way, it was an exception in the decrease of the percentage because it presented a prevalence increase of (6.5\%) comparing to the first 6 years of study. Conclusion - In this study it was observed a decrease of duodenal ulcer prevalence, $1.3 \%$ a year to be more accurate in 10 years of study, showing a statistical significance in the linear regression test.

HEADINGS - Duodenal ulcer, epidemiology. 


\section{REFERÊNCIAS}

1 Bobrzynski A, Beben P, Budzynski A, Bielánski W, Plonka M, Konturek S. Incidence of complications of peptic ulcers in patients with H.pylori infection and/or NSAID use in the era of Hp eradication. Med Sci Monit. 2002, 8:cr554-7

2 Chan FK, Leung WK. Peptic-ulcer disease. Lancet. 2002;360:933-41.

3 Chen TJ, Chou LF, Hwang SJ. Prevalence of anti-ulcer drug use in a Chinese cohort. World J Gastroenterol. 2003;6:1365-9.

4 Dore MP, Graham DY. Pathogenesis of duodenal ulcer disease: the rest of the story Baillieris Best Pract Res Clin Gastroenterol. 2000;14:97-107.

5 Graham DY, Klein PD, Opekun AR, Boutton TW. Effect of age on the frequency of active Campylobacter pylori infection diagnosed by the $13 \mathrm{C}$ Urea breath test in normal subjects and patients with peptic ulcer disease. J Infect Dis. 1988,157:777-80.

6 Gustavsson S, Nyren O. Time trends in peptic ulcer surgery, 1956 to 1986. A nationwide survey in Sweden. Ann Surg. 1989;210:704-9.

7 Heikkinen M, Pikkarainen P, Takala J, Julkunen R. General practioner's approach to dyspepsia. Survey of consultation frequencies, treatment and investigations. Scand J Gastroenterol. 1996;31:648-53.

8 Huang JQ, Sridhar S, Hunt RH. Role of Helicobacter pylori infection and non-steroidal anti-inflammatory drugs in peptic-ulcer disease: a meta-analysis. Lancet. 2002,359:14-22.

9 Kang JY, Tinto A, Higham J, Majeied. Peptic ulceration in general practice in England and Wales 1994-98: period prevalence and drug management. Aliment Pharmacol Ther. 2002;16:1067-74

10 Laine L. Nonsteroidal anti-inflammatory gastropathy. Gastrointest Clin N Am. 1996;6:489-504.

11 Laine L. Approaches to nonsteroidal anti-inflammatory drug use in the high risk patient. Gastroenterology. 2001,120:594-606.

12 Lanas A, Fuentes J, Benito R, Serrano P, Bajador E, Sainz R. Helicobacter pylori increases the risk ou upper gastrointestinal bleeding in patients taking low-dose aspirin Aliment Pharmacol Ther. 2002;16:779-86.

13 Lassen A, Hallas J, de Muckadell OB. Complicated and uncomplicated peptic ulcers in a Danish County 1993-2002: a population - based cohort study. Am J Gastroenterol. 2006, 101:945-53.

14 Loffeld RJ, van der Putten AN. Changes in the prevalence of Helicobacter pylori infection in two groups of patientsundergoing endoscopy and living in the same region in the Netherlands. Gastroenterol. 2003;38:938-41.

15 Marshall BJ, Armstrong JA, McGechie DB, Glancy RJ. Pyloric Campylobacter infection and gastroduodenal disease. Med J Aust. 1985,142:439-44.

16 Perez-Aisa MA, Del Pino D, Siles M, Lanas A. Clinical trends in ulcer diagnosis in a population with high prevalence of Helicobacter pylori infection. Alimen Pharmacol Ther. 2005,21:65-72.
17 Post PN, Kuipers EJ, Meijer GA. Declining incidence of peptic ulcer but not of its complications: a nation-wide study in the Netherlands. Aliment Pharmacol Ther. 2006;23:1587-93

18 Roosendaal R, Kuipers EJ, Buitenwerf J, van Uffelen C, Meuwissen SG, van Kampo GJ, Vanderbroucke-Grauls CM. Helicobacter pylori and the birthcohort effect: evidence of a continuous decrease of infection rates in childhood. Am J Gastroenterol. 1997;92:1480-82

19 Rosenstock S, Jorgensen T, Bonnevie O, Andersen L. Risk factors for peptic ulcer disease: a population based prospective cohort study comprising 2416 Danish adults. Gut. 2003;52:186-93

20 Sach G. Proton pumb inhibitors and acid - related diseases. Pharmacotherapy. 1997; 17:22-37.

21 Sakita T. Endoscopy in diagnosis of early gastric cancer. Clin Gastroenterol. 1973;2:345-60.

22 Sanchez-Bueno F, Marin P, Rios A, Aguayo JL, Robles R, Piñero A, Fernandez JA, Parrilla P. Has the incidence of perforated peptic ulcer decreased over the last decade? Dig Surg. 2001;18:444-7.

23 Schwesinger WH, Page CP, Sirinek KR, Gaskill HV 3rd, Melnick G, Strodel WE. Operations for peptic ulcer disease: paradigm lost. J Gastroint Surg. 2001;5:438-43.

24 Sonnemberg A, Everhart JE. The prevalence of self-reported peptic ulcer in the United States. Am J Public Health. 1996;86:200-5.

25 Susser M, Stein Z. Civilization and peptic ulcer. Lancet. 1962;1:115-9.

26 van Leerdam ME, Vreeburg EM, Rauws EA, Geraldts AA, Tijssen JG, Reitsma JB, Tytgat GN. Acute upper GI bleeding: did anything change? Time trend analysis of incidence and outcome of acute upper GI bleeding between 1993/1994 and 2000. Am J Gastroenterol. 2003, 98:1494-9.

27 Wolfe MM, Soll AH. The physiology of gastric acid secretion. N Engl J Med. 1988;319:1707-15.

28 Xia HH, Phung N, Antiparmak E, Berry A, Matheson M, Talley NJ. Reduction of peptic ulcer disease and Helicobacter pylori infection but increase of reflux esophagitis in Western Sydney between 1990 and 1998. Dig Dis Sci. 2001,46:2716:23.

29 Yuan Y, Hunt R. Treatment of non-NSAID and non-H pylori gastroduodenal ulcers and hypersecretory states. In : Therapy of digestive disorders. 2nd. Ed. 315-336 (Eds Wolfe MM et al) London, UK: Elsevier; 2006.

30 Yuang Y, Padol IT, Hunt R. Peptic ulcer disease today. Nat Clin Pract Gastroenterol Hepatol. 2006;2:80-9.

Recebido em 30/4/2007 Aprovado em 24/7/2007. 